\title{
The WORKPAD User Interface and Methodology: Developing Smart and Effective Mobile Applications for Emergency Operators*
}

\author{
Shah Rukh Humayoun ${ }^{1}$, Tiziana Catarci ${ }^{1}$, Massimiliano de Leoni ${ }^{1}$, Andrea Marrella ${ }^{1}$, \\ Massimo Mecella ${ }^{1}$, Manfred Bortenschlager ${ }^{2}$, and Renate Steinmann ${ }^{2}$ \\ ${ }^{1}$ Dipartimento di Informatica e Sistemistica "Antonio Ruberti" \\ SAPIENZA - Università di Roma \\ Via Ariosto 25, Roma, 00185, Italy \\ ${ }^{2}$ Salzburg Research forschungsgesellschaft $\mathrm{mbH}$ \\ Jakob-Haringer-Str. 5/III, 5020 Salzburg, Austria \\ \{humayoun, catarci, deleoni, marrella, mecella\} ddis.uniromal.it, \\ \{manfred.bortenschlager, renate.steinmann\} @salzburgresearch.at
}

\begin{abstract}
In complex emergency/disaster scenarios, teams from various emergency-response organizations collaborate in order to achieve a common goal. The use of smart mobile devices and applications in these scenarios can improve this collaboration dynamically; and poses interesting challenges, such as user' mental attention, small screen size, unavailability of reliable network, reduced power, and battery consumption. So, to design and develop interactive applications to be used in mobile and pervasive scenarios requires novel methodologies which combine user-centred design approaches and software engineering approaches tailed for distributed architectures. In this paper, we outline the methodology, adopted successfully in the European WORKPAD project, and describe the work done from getting the requirements to developing the interface of the desired system.
\end{abstract}

Keywords: Emergency Scenarios, Mobile Systems, User Interface, UserCentred Design, Requirement Collection.

\section{Introduction}

The term "emergency management" means the coordinated activities both to prevent disaster happenings and to face them when they take place. Such activities consist of five phases [7]: planning, mitigation, preparedness, response, and recovery. The European project WORKPAD (http://www.workpad-project.eu/) provides a software and communication infrastructure to support operators in emergency scenarios, by focusing on the most critical phases i.e., the response and the short-term recovery.

* This work has been partly supported by the EU Research Project WORKPAD http: / /www. workpad-project.eu/ 
In disaster scenarios, different teams, belonging to different organizations, need to collaborate in order to reach a common goal. So the collaboration within teammembers and with other teams operating at the disaster site(s) is very critical, as the achievement of the desired goal heavily depends on this collaboration and coordination. The use of mobile devices and applications in these scenarios is very valuable as they can improve collaboration, coordination, and communication amongst team(s) to achieve the desired goals. But there are also risks involved while using these mobile applications, e.g., decreasing of performance. Secondly, in emergency/disaster scenarios, most of the tasks are highly critical and time demanding; for instance, in these scenarios saving minutes could result in saving people's life. Therefore, it is unacceptable to use systems that lack proper interaction principles. In WORKPAD, to cope the challenges of such critical environment, we adopted a two-fold methodology (bottom-up and top-down) with focus on user-centred design (UCD) principles to develop an adaptive peer-to-peer (P2P) software and communication infrastructure. This adopted approach of continuously involving real-end users for getting requirements and designing systems is the main difference between WORKPAD project and the previous research projects for emergency management.

The rest of the paper is structured as follows: Section 2 outlines very briefly the adopted methodology and the activities that were conducted for the WORKPAD project. Section 3 describes how we developed the user interface of smart mobile devices for emergency operators. Section 4 provides the details about the conducted usability evaluation tests. The paper concludes with Section 5.

\section{The WORKPAD Methodology for Developing Mobile Applications in Emergency Scenarios}

In the WORKPAD project, a two-fold (bottom-up and top-down) high-level approach with various HCI (Human-Computer Interaction) techniques was selected for taking the requirements and for designing the system. The work done according to the selected approach is as follow:

- Bottom-up approach: A concrete case study of emergency management in the Calabria region was conducted. Potential users were intensively involved in this project phase according to the international ISO standard 13407 (Human-Centred Design Processes for Interactive Systems) [2].

- Top-down approach: On the one hand we investigated European legislation, recommendations, and initiatives with respect to emergency management, and on the other hand, related European research projects were examined regarding the adopted requirements analysis methods, the concrete outcomes and their validity for the WORKPAD project.

\subsection{System Engineering Methodology by Using a User-Centred Design (UCD) Approach}

In WORKPAD, an iterative and incremental approached was developed, in order to reduce the risks of functionality/usability failure of the project prototype through steady 


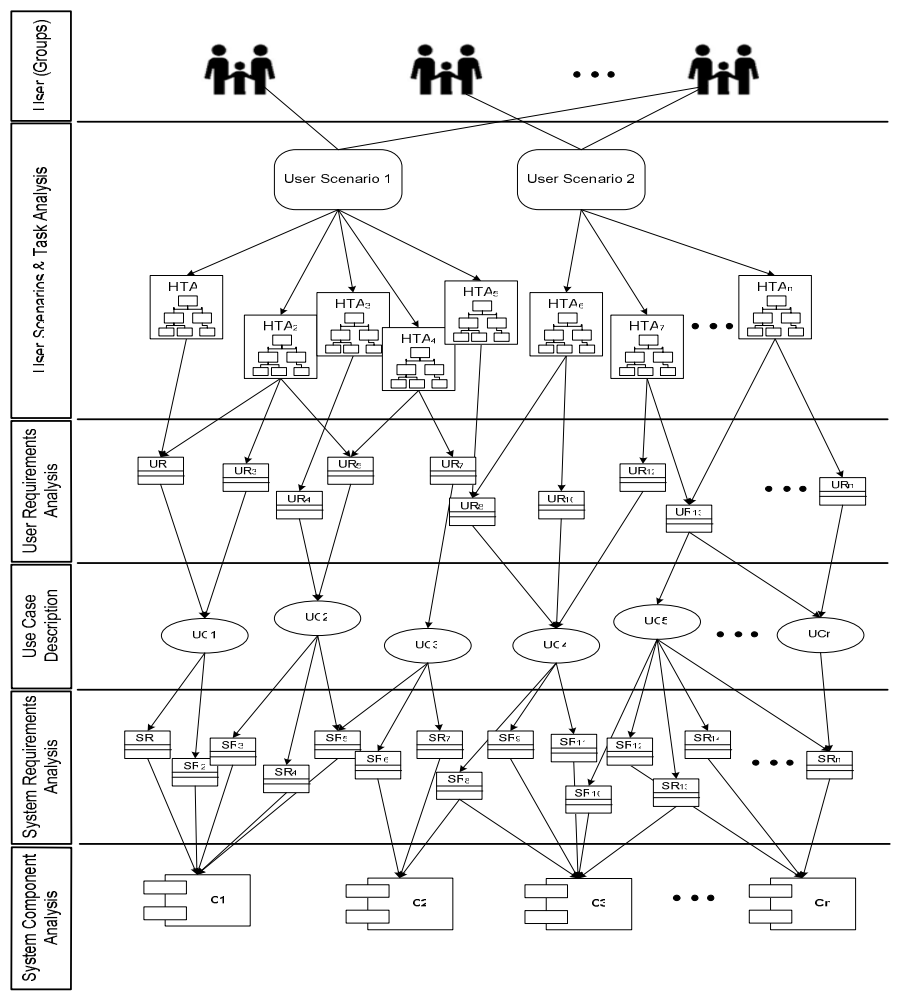

Fig. 1. WORKPAD system engineering methodology

feedbacks with the users. Figure 1 gives a technical overview of the methodology used for the practical parts of the requirement elicitation process, and depicts several phases and their interrelations. These phases are comprised of the definition of user groups, development of scenarios, task analysis, requirement derivation, use case definition, system requirement analysis, and finally the analysis of the required WORKPAD system components. A detailed description of each phase can be found in [6].

\subsection{The WORKPAD Project: A Case Study}

The first task conducted during the case study of Calabria was to identify the potential users and user groups. We interviewed some officers and actors which are actively involved during emergencies and identified two typologies of users: front-end users who act directly in the field during emergencies/disasters (ranging from firemen to voluntary associations); and back-end users who manage the situation from control rooms, by providing goals/instructions/information to front-end operators.

In order to learn users, with their problems and expectations, first we conducted semi-structured interviews using open-ended questions. We conducted 32 interviews, each approximately 45 minutes long, from officers and generic actors of the most important organizations involved in emergency management in the Calabria region. 
We concentrated on two scenarios: earthquake and flood, and designed activity diagrams for both scenarios to describe how the operators would follow in order to face the emergency situation. Due to the necessity of more detailed data, we conducted 14 further targeted user interviews to refine the proposed storyboards.

In order to go deeply into "the mind" of rescue operators, we asked them to illustrate their own personal experiences in past-occurred disasters, called storyboards. Using these storyboards, we conducted a task break-down analysis through classical Hierarchical Task Analysis (HTA) [1] technique. In parallel, more general requirements were identified and analysed by examining several national and international regulations and legislations, e.g., through the Vade mecum of Civil Protection in the European Union [6] and the International Civil Defence Directory [4], and additionally by investigating related research projects.

As depicted in Figure 1, user requirements serve as input for the use cases, through UML use case diagram, and system requirements are the outputs. The overall set of the resulting system requirements were clustered according to the functional entities of the intended WORKPAD system. The system component analysis, as the last stage, is the interface to the concrete design and implementation tasks and work-packages.

\subsection{Related Research Projects}

In recent years, many projects and initiatives, especially in Europe, have investigated issues related to the ones considered by the WORKPAD project. Few examples of them are AMIRA (http://www.amira.no/), WIN (http://www.win-eu.org/), OASIS (http://www.oasis-fp6.org/), and LIAISON (http://1iaison. newapplication.it/liaison/). Table 1 confronts the analysed projects and the deployed requirements capturing methods.

Table 1. Comparison of user methodology of related EU research projects

\begin{tabular}{|l|c|c|c|c|}
\hline & $\begin{array}{c}\text { Review of former } \\
\text { projects }\end{array}$ & $\begin{array}{c}\text { Review of publicly } \\
\text { available documents }\end{array}$ & Interviews & $\begin{array}{c}\text { Second interview } \\
\text { iteration }\end{array}$ \\
\hline AMIRA & Yes & yes & Yes & no \\
\hline LIAISON & No & no & Yes & no \\
\hline OASIS & Yes & yes & Yes & yes \\
\hline WIN & Yes & no & Yes & yes \\
\hline
\end{tabular}

By the analysis of the methodologies used in these projects, apparently, there comes out the result that all these investigated projects adopted nearly similar methods to collect requirements: end-user interviews in addition to reviews of previous related works. In WORKPAD project, besides the theoretical examination of the related work, we also worked together as closely as possible with real-end users (the case study of Calabria region). Moreover, our methodology is deeply based on the UCD approach adopted from ISO 13407 [2] so to deploy iteratively other HCI techniques such as scenarios, focus group meetings, storyboards, and task analysis. This is the main difference between WORKPAD methodology and the methodologies used in these related projects. 


\section{The WORKPAD User Interface Construction}

The teams/operators working in disaster/emergency situations will use smart mobile devices in very dynamic and mobile scenarios over a network partially unreliable. Therefore, some challenging issues emerge, which we divided into two categories.

- The first category concerns grasping the users' mental attentions onto the system as little as possible because pervasive processes are really challenging and stressing for them.

- The latter category of issues is merely technological and deals with reducing the resource consumptions.

The following subsections give a brief overview of the WORKPAD architecture followed by the steps taken to tackle the above categories of challenges during the construction of the user interface.

\subsection{The WORKPAD Architecture}

According to the WORKPAD architecture, several teams comprise the system frontend: team members belong to the same organization (e.g., the fire department) and carry smart mobile devices (such as PDAs or smart phones). They establish a mobile ad hoc network (MANET) for coordination and intra-teams communication. The lack of a fixed infrastructure makes this kind of network suitable in emergency management/disaster scenarios. The WORKPAD back-end is a peer-to-peer (P2P) overlay network that includes the operating organization's back-office systems (such as services and databases). Front-end operators access the back-end network through their back-office systems. There, they can get or set the information that is relevant to their situation or planned action.

\subsection{Cognitive and Ergonomics Issues}

When designating the user interface of a system, the first aspect to consider is how and where users address their mental attention. The human beings receive continuously a huge quantity of stimuli from the environment. In [5], attention is defined as the totality of information cognitively manipulated by a person. The attention allows human beings to consider stimuli in a judicious way, prioritizing them, and taking into account only the most important ones. This judiciousness is used to increase the probability of a rapid and accurate answer.

Activities in critical and emergency scenarios are highly-stressing situations for the users, who generally give more priority on the physical stimuli concerning the activities to execute than on those coming from software applications. Therefore, when designing client interfaces for mobile, pervasive, and critical scenarios, it is important that the task handling interface should attract the user' attention only when it is strictly required. In few words, we want the system to act as an automatic process for the users, which scarcely need for cognitive resources when using the system. Another aspect worthy to consider is accessibility and ergonomics issues when using PDAs in critical emergency scenarios. 


\subsection{Designing and Constructing the WORKPAD User Interface}

The first step in designing the user interface was to understand how to organize the needed information in screens that have a reduced size, such as PDA; this is a critical issue, as the operator should quickly access a lot of information, whereas the dimension of the screen could cause this retrieval to take several steps. Moreover, during an action it is usual that an operator, whose attention is completely turned to the task assigned to him/her, can forget the exact arrangement of the information items on the screen. Therefore, he/she should be able to recover it in his/her mind the arrangement of the items through a fast glance from the PDA's screen. As already widely studied and demonstrated (see for example [3]), the maximum number of items that a subject can store instantaneously in a reduced time (approximately 200-300 milliseconds) is about 4 items. This categorization brought us to divide the available information in 2 or 3 macro-categories. These macro-categories can be easily accessed through the use of tabs on the left side of screen (see Figure 2), without filling the screen with huge set of objects.

From several studies on the psychology of users, which employ systems, they, when using interfaces, tends not to read whole words but only some letters in order to understand their meanings. So, we designed WORKPAD user interface with familiar formats and fonts, which are also big enough. That allows operators to remind word easily.

Another important step was to understand how to capture the operators' attention while they were carrying out tasks. For this, we have made a significant use of popups and sonorous alarms to achieve the results. Considering the accessibility and ergonomics issues, we have taken into account the fact that these devices may be used in extreme conditions. So, particular precautions were taken when designing the user interface. In particular, an effective and easy-to-read choice of colours; the highly contrasting colour chosen in order to be clearly visible in particular light conditions (e.g., in night missions). Moreover, the interaction with the interface takes mostly place through fingers, instead of the stylus. Therefore, the user interface elements were sized and spaced out in order to avoid the users to press on wrong elements because they are close to those that the users were willing to push.

On the technical side, when devising the system we kept in mind to reduce as much as possible the use of three kinds of resources: the computational power, the bandwidth, and the battery that are quite limited for smart devices.

Figures 2 shows few screen shots of the Task Handler of WORKPAD user interface of the front-end application. Figure 2(a) shows the screen shot of the Task Handler of the team leader while picking the capabilities he/she can cover from a check-box list. Figure 2(b) shows the task Handler of a team member after assignment of a task to her. Figure 2(c) shows the Context Editor of the Task Handler that is meant to fill in a certain questionnaire for the assessment, while $4(d)$ shows the actual implementation of the feature that allows the team leader to gain an insight of the status of all team members. 


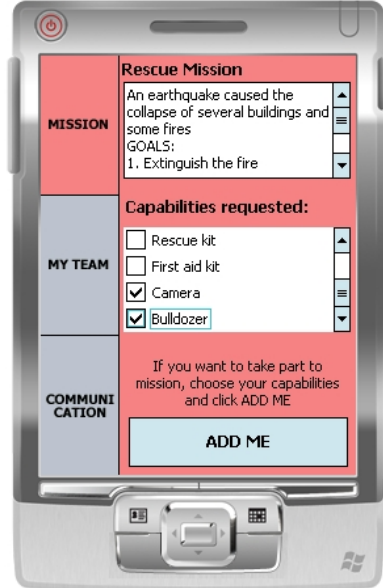

(a) Task Handler from the team-leader's PDA

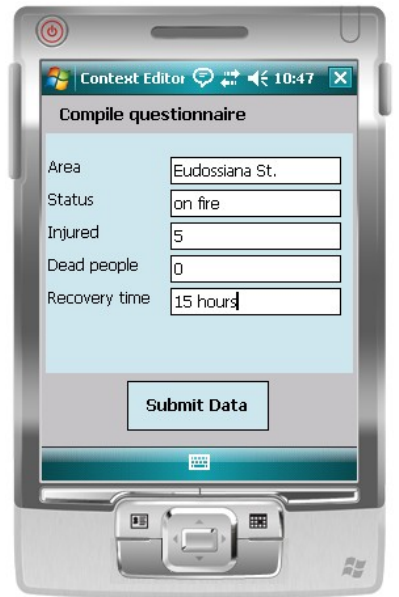

(c) Context Editor of the Task Handler

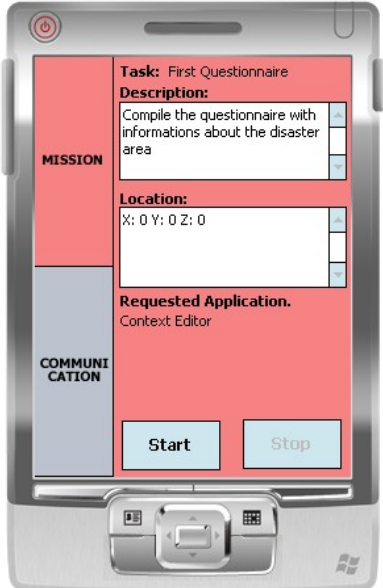

(b) Task is assigned to a team-member

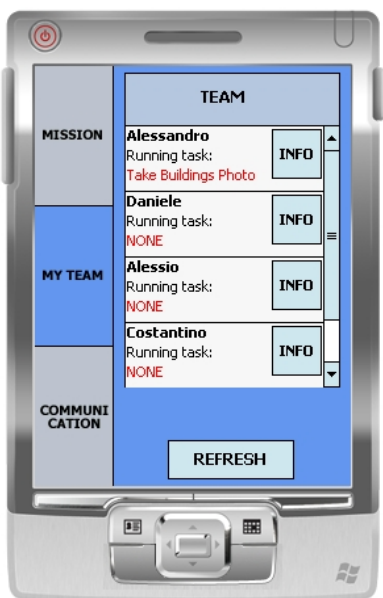

(d) The status of all team members on team leader's device

Fig. 2. Some screen shots of the WORKPAD Task Handler interface

\section{The WORKPAD Usability Evaluation}

The WORKPAD usability methodology is based on UCD approach adopted from ISO 13407 [2], which means end-users are actively involved in the usability evaluations that are performed throughout the whole project lifecycle to ensure an interactive and an easy-to-use system. We planned an evaluation and validation roadmap along the project development life-cycle. We have already performed two usability tests, mainly using qualitative usability evaluation methods like feature inspection or observation of users, with selected users from the Calabria Homeland Security Department and one from the external users to ensure the user-driven development. 
These user tests were analysed and served as recommendation inputs for the system designers and developers. In early 2009, we are performing a controlled experiment to test and compare WORKPAD user interface under controlled experiment conditions, and at the end of the project, we will implement and evaluate the "WORKPAD Showcase" that represents the implementation of a concrete emergency management scenario in a real world context. The following subsections provide the details of usability tests that we already have conducted.

\subsection{Pre-test with Online Mock-Ups}

We performed a pre-test in the second phase of the project, when mock-ups of the components were available in digital form and ready for testing with potential users, while using online questionnaire for online evaluation. There were 13 users ( 8 male and 6 female) from Calabria region, 3 were from 46-60 age range and 10 were from 31-45 age range. Six users were inexperienced in using a PDA, while 2 were very experienced users. Few results from the mock-up pre-test are:

- Most of the participants were agreed that all WORKPAD components are understandable and intuitive.

- 8 users agreed and 5 users partially agreed that the Task Management component helps them in performing different tasks in the case of an emergency.

- 12 users said that it is comprehensive to perform a task by undertaking different steps.

- 10 users agreed that the instructions and the buttons are clearly formulated.

- 10 users considered the chosen symbols as appropriate, while the one said it was very appropriate.

These are few comments and feedbacks that we received from participant users as recommendations for improvement in the interface such as: usage of textual commands or description beside icons; predefined inputs (e.g., drop-down lists); toolbars with frequently used functions represented by an icon, help guides (e.g., tool-tips); enabling zoom-in and -out, coordinates and scale in Map Overview component; capturing and sharing of actual emergency situation picture.

\subsection{Second Usability Testing with Real Prototype Using Cooperative Evaluation Methods}

The second usability testing was performed near out field test site Pentedattilo in Calabria region during July 2008. There were 4 participant users involved, all male and below of age 30, from PCRC organization "Le Pantere Verdi", and 16 further interested potential users were present. Among participant users, 2 were much experienced, 1 was normal, and the remaining one was inexperienced in using PDAs. We performed a cooperative evaluation with the first real prototype in two parallel sessions with one user each of about one and half hour long. Each user performed several tasks with the different WORKPAD components, while they have been recorded audio and video. Table 2 shows the results of this test. 
Table 2. WORKPAD prototype testing with emergency operators users

\begin{tabular}{|l|c|c|c|c|}
\hline & $\begin{array}{l}\text { Fully } \\
\text { Agree }\end{array}$ & $\begin{array}{l}\text { Partially } \\
\text { Agree }\end{array}$ & $\begin{array}{l}\text { Partially } \\
\text { Disagree }\end{array}$ & Disagree \\
\hline Components are intuitive and easy to use & 3 & 1 & $\mathrm{X}$ & $\mathrm{X}$ \\
\hline Components run without any interruptions and crashes & 3 & 1 & $\mathrm{X}$ & $\mathrm{X}$ \\
\hline Screen design is attractive & 2 & 2 & $\mathrm{X}$ & $\mathrm{X}$ \\
\hline Screen text is easy to read & 2 & 2 & $\mathrm{x}$ & $\mathrm{X}$ \\
\hline Components are easy to navigate & 2 & 2 & $\mathrm{x}$ & $\mathrm{X}$ \\
\hline $\begin{array}{l}\text { WORKPAD supports in performing tasks in the case } \\
\text { of an emergency }\end{array}$ & 3 & 1 & $\mathrm{x}$ & $\mathrm{X}$ \\
\hline
\end{tabular}

From the table, it can be summarized that we improved very much the usability level of WORKPAD user interface from the results and recommendations of first usability testing. Few comments and suggestions for improving design were: avoiding of long scrolling times in the Context Editor component; significant usage of colours (e.g., red for emergency, yellow for fir and so on); usage of PCRC colour standard; bigger size of map in the GIS Client component.

\subsection{Usability Testing with External Users}

We have also performed a usability testing with "external" users (e.g., colleagues, friends, etc.) who were not from the emergency management. Different partners, responsible for developing WORKPAD user interface, contributed in this test with 4 to 6 people on each site. 21 external users (14 male and 7 female); belonging to Austria, Italy, and Czech Republic were involved. Among them, 7 were very experienced, 7 were not at all, 2 were much experienced, and the remaining 7 were not so much experienced with using PDAs. We tested each component with a group of 4-6 external users. Among them, 13 agreed and 6 disagreed that their component is intuitive and easy to use. Table 3 shows the results, where each row shows the total number of participants that gave their satisfaction level of their tested component. In few cases, few participants did not give any feedback.

Table 3. WORKPAD prototype testing with external users

\begin{tabular}{|l|c|c|c|c|}
\hline & $\begin{array}{l}\text { Fully } \\
\text { Agreed }\end{array}$ & $\begin{array}{l}\text { Partially } \\
\text { Agree }\end{array}$ & $\begin{array}{l}\text { Partially } \\
\text { Disagree }\end{array}$ & Disagree \\
\hline $\begin{array}{l}\text { Component runs without any interruptions and } \\
\text { crashes }\end{array}$ & 13 & 4 & 2 & 2 \\
\hline Screen design is attractive & 7 & 11 & 1 & 1 \\
\hline Screen text is easy to read & 7 & 11 & 3 & $\mathrm{x}$ \\
\hline Component is easy to navigate & 8 & 8 & 4 & $\mathrm{x}$ \\
\hline The screen design is consistent & 10 & 8 & 2 & $\mathrm{x}$ \\
\hline
\end{tabular}

\section{Conclusion}

A smart and effective system for mobile devices, with well-focused interaction style, can play a critical role in achieving goals for the team/s working in emergency/disaster situations. It can improve the collaboration, coordination, and communication amongst 
team members who work in such highly critical scenarios. For developing these smart and effective applications for mobile scenarios, there is need to develop novel approaches that cope the challenges of these critical environments. In this paper, we present our methodology that we used to design and to develop the European project WORKPAD, which provides an adaptive peer-to-peer (P2P) software and communication infrastructure to support human operators in emergency/disaster scenarios. The continuous involvement of real-end users for getting the requirements and to design the system is the main difference between the WORKPAD project approach and the previous research projects for emergency management. Currently, we are also successfully applying the same adopted methodology (two-fold with focus on UCD principles) after further refinements in other projects.

\section{References}

1. Dix, A., Finlay, J., Abowd, G., Beale, R.: Human Computer Interaction, 3rd edn. PrenticeHall, Englewood Cliffs (2003)

2. International Standardisation Organisation: Human-Centred Design Processes For Interactive Systems. International Standards Organization, ISO Standard 13407 (1999)

3. Luck, S.J., Vogel, E.K.: The Capacity of Visual Working Memory For Features And Conjunctions. Nature 390, 279-281 (1997)

4. International Civil Defence Directory: Activities International Cooperation, http: / / www. icdo.org/act-coopdirectory. html (last accessed, 11.4.2007)

5. Sternberg, R.J.: Cognitive Psychology, 3rd edn. Wadsworth Publishing (2002)

6. Humayoun, S.R., Catarci, T., de Leoni, M., Marrella, A., Mecella, M., Bortenschlager, M., Steinmann, R.: Designing Mobile Systems in Highly Dynamic Scenarios. The WORKPAD Methodology. Journal on Knowledge, Technology \& Policy 22(1) (2009)

7. European Council (2001): Council Decision of $23^{\text {rd }}$ of October 2001, Establishing A Community Mechanism To Facilitate Reinforced Cooperation In Civil Protection Assistance Interventions (2001/792/EC, Euratom). Official Journal of the European Union, 7-11 (2001) 